\title{
A review of non-strabismic accommodative and vergence anomalies in school-age children. Part 2: Accommodative anomalies
}

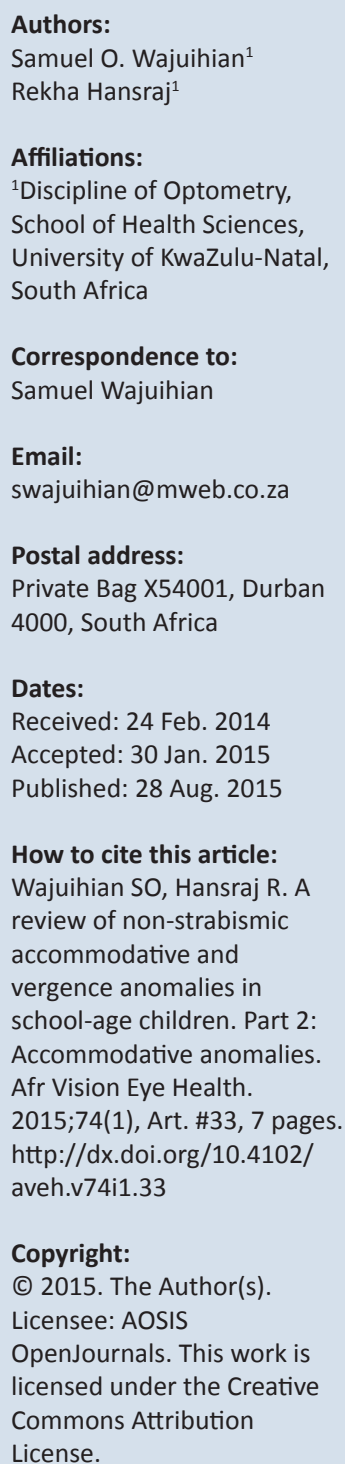

Copyright:

(C) 2015. The Author(s).

Licensee: AOSIS

OpenJournals. This work is licensed under the Creative Commons Attribution License.

\section{Read online:}

Comfortable reading and the performance of related near point activities involve efficient accommodative and vergence systems. However, accommodative and convergence anomalies are associated with various symptoms of asthenopia that impair efficient near point tasks. In Part 1 of this two-part article, studies on vergence anomalies were reviewed. In the current paper (Part 2), anomalies of accommodation are reviewed. The aims of the latter paper were to derive the prevalence and distribution estimates of anomalies of accommodation in schoolage children and address variations in the study methods and findings. Despite variations in the study methods and findings, anomalies of accommodation are prevalent among school-age populations. Variations and limitations of previous studies are discussed and recommendations for improving future studies are suggested.

\section{Introduction}

Accommodative and vergence binocular anomalies are vision disorders that affect clarity and binocularity, and impair comfort and efficiency of visual performance of an individual when near tasks such as reading, writing and computer-based works are performed. ${ }^{1,2,3,4,5}$ For the school-age child, especially the high school learner, symptoms associated with accommodative-vergence anomalies tend to increase as the child advances through school; $1,2,3,4,5$ this is because there is a greater demand at higher grade levels on the accommodative and vergence system for sustained clear vision owing to prolonged reading and increased information processing. ${ }^{1,2,3,4,5}$ In Part 1, studies on vergence anomalies in school-age children were reviewed. In the current paper (Part 2), anomalies of accommodation are reviewed.

\section{Methods and scope of review}

The following electronic databases were searched using the terms accommodative insufficiency, accommodative excess and accommodative infacility: PubMed, Medline, Science Direct, Google Scholar, EBSCO and Embase. Relevant articles on reference lists were identified and retrieved from electronic or print journals. Manual searches of tables of contents of print and electronic versions of optometry journals at the University of KwaZulu-Natal Library were also conducted and interlibrary loan facilities were utilised where necessary. Over $90 \%$ of the reviewed articles were obtained from manual identification on reference lists of published papers. An article was considered for review if it reported on the epidemiology (prevalence and distribution) of accommodative anomalies, was a primary research article published in a peer-reviewed journal, and was in the English language.

For the review, studies were first profiled individually, followed by comparisons and synthesis with other studies on various aspects of study design and findings. The sources of variations and limitations of studies are discussed. The review is of school-age children between 6 and 18 years, and only original papers that focused on epidemiology (prevalence and distribution) of anomalies of accommodation were considered for the main review. A few classic papers from the early $1990 \mathrm{~s}^{6,7}$ were included to relate previous concepts to current understanding. Summary tables for the anomalies of accommodation are presented.

\section{Accommodation parameters and previous studies on accommodative anomalies}

Accommodation is a process by which the dioptric power of the eye is changed to obtain clear retinal images when focusing on an object closer than infinity. ${ }^{8,9}$ The stimulus to accommodation is a blurred retinal image, ${ }^{10}$ and the accommodative process is mediated by a contraction 
TABLE 1: Symptoms in accommodative anomalies. ${ }^{8,12,13,19,28}$

\begin{tabular}{ll}
\hline Number & Symptoms \\
\hline 1 & Blurred vision for near tasks \\
2 & Headaches/eyestrain/dull orbital pain/pulling sensation around the eyes \\
3 & Loss of comprehension/avoidance of near work \\
4 & Watering or conjunctival or eyelids irritation, sensitivity to light \\
5 & Eye fatigue/dizziness/sleepiness \\
6 & Blurred vision worse after reading/near work \\
7 & Difficulty focusing from far to near, or near to far \\
8 & Holds reading material close or farther way \\
9 & $\begin{array}{l}\text { Difficulty sustaining attention on near point tasks; distance blur after } \\
\text { performing near work }\end{array}$ \\
\hline
\end{tabular}

or relaxation of the ciliary muscles. ${ }^{10}$ This process of accommodation results in an increase or decrease of the curvature and thickness of the crystalline lens, leading to a change in the refractive power of the eye. ${ }^{10}$ An ability to accommodate rapidly, smoothly, accurately and sustain focus for the required time interval is important for a child to perform near tasks efficiently. ${ }^{11}$ The accommodation mechanism is extremely important for reading and learning, as children who suffer anomalies of accommodation are more prone to fatigue quickly and become inattentive than those who have normal accommodation functions. ${ }^{11}$

In accommodative anomalies, the eyes are unable to focus clearly and efficiently on objects at varying distances, which may result in unclear retinal images with characteristic symptoms. ${ }^{8,12}$ The symptoms in accommodative anomalies (Table 1) are interrelated, although each syndrome may have unique symptoms. ${ }^{8,12,13}$

\section{A review of previous studies on accommodative anomalies}

Although it is useful clinically to separate anomalies of accommodation into distinct entities, there may be no marked boundaries between the subgroups of accommodative anomalies. ${ }^{12}$ Accommodative disorders are more significant clinically when diagnosed as syndromes, ${ }^{12}$ and reports on original studies reviewed were based on the three main syndromes: accommodative insufficiency (AI), accommodative excess (AE) and accommodative infacility (AIF). Several studies ${ }^{12,13,14,15,16,17,18,19,20,21,22,23,24,25,26,27}$ have reported on various aspects of accommodative anomalies and in various racial groups, populations and study settings.

\section{Accommodative insufficiency}

The term accommodative insufficiency concerns paralysis of, and ill-sustained and unequal, accommodation. ${ }^{6,7,8,12}$ However, both paralysis of accommodation (complete loss of accommodative function where the use of the eyes for near tasks is impossible), and paresis (partial loss of accommodative function) are typically the result of either toxins or lesions of the nervous system. ${ }^{8,28}$ Accommodative insufficiency is an anomaly that is characterised by an inability to focus or sustain focus at near, diagnosed clinically by insufficient amplitude of accommodation (AA) that is below the lower limit expected for the person's age based on age-expected norms. ${ }^{6,8}$ The direct clinical signs for diagnosing AI include low AA, high monocular estimation method (MEM) retinoscopic findings and difficulty in clearing -2 D lenses with monocular accommodative facility (MAF). ${ }^{8}$ The techniques used to diagnose $\mathrm{AI}$ in earlier studies were similar and included mainly push-up-to-blur technique (PUBT), MEM and \pm 2 D lens flippers. The PUBT was applied in all studies reviewed except one study ${ }^{18}$ that used the PUBT and push-down techniques to measure AA. AI has been reported to be the most common accommodative anomaly and a common cause of asthenopia in school-age children., ${ }^{8,28}$

The findings from studies are diverse mainly because of variations in criteria applied to define the anomalies; consequently, one approach that was applied to facilitate comparison of the findings was to present the review based on the type of population from which the samples were derived, such as non-clinical ${ }^{14,15,16,19,20,21,22,23}$ or clinical. ${ }^{17,24,25} \mathrm{~A}$ second approach was to classify studies based on the number of clinical signs applied to define AI (single or multiple clinical signs). With the single criterion, AI was defined as $2 \mathrm{D}$ below Hoffstetters' formula for minimum AA ( $=15-0.25$ age) and was applied by various studies. ${ }^{14,15,18,19,20,24,26,27}$ With multiple signs or criteria, the single criterion is applied in addition to other clinical signs. ${ }^{13,17,22,23}$

\section{Studies on non-clinical populations}

Studies on non-clinical populations were conducted mainly in school settings. Such studies enable random sampling, improve generalisations of findings and offer the merit of testing children in environments similar to those in which they learn. Studies on non-clinical populations include Borsting et al. ${ }^{14}$ who studied accommodative and convergence insufficiency in schoolchildren aged between 8 and 15 years. Participants were recruited from four schools. AA was measured using the push-up technique and defined using the single criterion. The prevalence of AI was $17 \% .^{14}$ Using similar techniques and criteria, Marran et al. ${ }^{15}$ studied accommodative and convergence insufficiency in relation to symptoms in 299 schoolchildren (mean age 11.5 years). The study participants were randomly selected from 19 elementary schools. The prevalence of AI was $4.7 \%$ and more girls than boys had AI. ${ }^{15}$ Given that both studies applied similar techniques and criteria, a possible source of difference between both studies ${ }^{14,15}$ might be related to subjectiveness of the measurement techniques.

Three studies $19,20,21$ with Swedish schoolchildren measured AA using the PUBT and defined AI using the single criterion. In 2004, Sterner et al. ${ }^{19}$ studied AA in 76 elementary schoolchildren aged between 6 and 10 years. The schools were randomly selected although the relatively small sample size and non-random selection of participants within schools for the study might have limited generalisation of the findings. The prevalence of AI (reported as an average) was 34\%. ${ }^{19}$ Remarkably, Sterner et al. ${ }^{19}$ found no correlation between age and monocular or binocular accommodation and recommended that AA be routinely evaluated at all ages as 
it may be misleading to assume that younger children always will have maximum AA. In 2005, Abdi and Rydberg ${ }^{20}$ studied asthenopia and binocular functions in 120 Swedish children aged between 6 and 16 years. The prevalence of AI (classified as 'moderate and marked') was $24.2 \%$. The participants in the study were symptomatic children who were referred for eye care by their school nurses, and the expected prevalence might have been overestimated. Abdi et al. in $2008^{21}$ studied orthoptic functions in 216 Swedish schoolchildren aged between 6 and 16 years, and found that $11.1 \%$ of the participants had AI and that the frequency of accommodative anomalies for children in higher grades was greater than those in lower grades. This finding supports the fact that accommodative anomalies increase with age. An increased prevalence of AI with age may also be related to increasing near task demands with higher levels of school grade.

Taken together, the differences in findings for the studies that defined AI using the single criterion might be because of the following factors. Abdi and Rydberg ${ }^{20}$ studied children with asthenopic symptoms who had problems with their school work and were referred for eye examination. Sterner et al. ${ }^{19}$ proposed that a possible reason for the high prevalence they found is that children who had near vision problems might be more willing to respond to an invitation to participate in the study. Similarly, the participants in Abdi et al.'s study ${ }^{21}$ were based on participants' invitation. Consequently, the sample selection strategy might have influenced the findings from these studies. ${ }^{19,20,21}$ Furthermore, being a subjective technique, young children might interpret the end points poorly, which might bias the findings.

Shin et al. ${ }^{22}$ in 2009 investigated accommodative and vergence anomalies in 216 South Korean schoolchildren aged from 9 to 13 years. AA was assessed using the PUBT, and AI was defined using multiple criteria. The reported prevalence of AI was $18.3 \%$. Shin et al. ${ }^{22}$ applied multiple criteria to define $\mathrm{AI}$ and reported a relatively high prevalence of $18.3 \%$. The finding of a relatively high prevalence of AI suggests that race may be a contributory factor to the differences in findings compared with other studies, as race has been reported to influence accommodation parameters. ${ }^{29}$ The study by Shin et al. $^{22}$ was conducted on Asian schoolchildren whereas the other studies ${ }^{14,15,19,20,21}$ were on white populations. From the reviewed studies, the influence of gender on AI appears inconclusive as only the study by Marran et al..$^{15}$ reported on the gender distribution of $\mathrm{AI}$ and found that more female than male subjects had AI. In a pilot study comprising 65 high school learners, Wajuihian and Hansraj ${ }^{23}$ reported a prevalence estimate of $1.6 \%$ for AI.

\section{Studies on clinical populations}

The main technique for the studies in this category was PUBT, and either single or multiple-sign criteria were applied to define AI. In 1995, Dwyer ${ }^{24}$ studied 'vergenceaccommodative' disorders in 144 patients aged between 7 and 18 years. Participants for the study were consecutive patients who were examined at the author's optometry practice in
Australia. Dwyer ${ }^{24}$ reported that only one participant (0.7\%) had AI. Similarly, for children aged from 6 to 14 years, Dusek et al. ${ }^{25}$ reported a prevalence of $0.6 \%$ for schoolchildren who attended an optometric practice in Austria. In 1996, Scheiman et al. ${ }^{17}$ investigated vision and ocular anomalies in 2023 consecutive patients who attended the Pennsylvania College of Optometry clinic. The ages of patients ranged between 6 months and 18 years (although 1650 children were aged between 6 and 18 years). The prevalence of AI was 2.3\% for school-aged children and $0.8 \%$ for preschool children, which indicates an increase in AI prevalence with age. ${ }^{17}$

Other studies ${ }^{11,16,18,26,27}$ did not specify a diagnosis of AI but reported on subnormal or poor AA which was based on the single criteria of 2 D below Hoffstetter's minimum age formula; therefore a single-criterion diagnosis of AI may be inferred. In 1985, Helveston et al. ${ }^{16}$ studied visual functions and academic performance in 1910 first-, second- and thirdgrade schoolchildren in the USA. Although the study can be credited with a relatively large sample size, a possible limitation is that the diagnostic criteria were arbitrarily chosen by the examiners on the basis of the authors' previous clinical experience, without references to published criteria. Helveston et al. ${ }^{16}$ reported that between $7 \%$ and $10 \%$ of the participants had subnormal AA. Another study in a clinical population was reported by Benzoni and Rosenfield ${ }^{18}$ who studied AA in 60 asymptomatic children between 5 and 10 years old who attended a university optometry clinic in the USA. Both the push-up and push-down techniques were used to assess AA. Benzoni and Rosenfield ${ }^{18}$ reported that $36 \%$ of the participants had AA of $2 \mathrm{D}$ below Hofstetter's minimum value and that AA declined with increasing age. In the USA, Wick and Hall ${ }^{11}$ studied accommodative functions in 1987 in 200 elementary schoolchildren from grades one through six. The PUBT was used to assess AA although the binocular amplitude was used instead of the monocular to calculate reduced AA in Hofstetter's formula. Wick and Hall ${ }^{11}$ found that $25 \%$ of the children had amplitudes below their expected age minimums. The findings may be difficult to interpret as Hofstetter's formula is based on monocular amplitude. A shortcoming of this approach is that binocular AA may mask monocular anomalies and is often higher than monocularly determined AA owing to the influence of vergence-induced accommodation. ${ }^{19,30}$

Two studies ${ }^{26,27}$ in South Africa, which both measured AA using the PUBT, reported on accommodative functions. Metsing and Ferreira ${ }^{26}$ studied accommodative and vergence status amongst 80 primary school children aged between 8 and 13 years. Seven of the learners were excluded from the study owing to poor subjective responses. Using Hofstetter's formula, the authors reported that $10 \%$ of the participants had poor monocular AA. Moodley ${ }^{27}$ conducted a retrospective report of a vision screening of 264 schoolchildren aged between 6 and 13 years in Durban, South Africa. Moodley ${ }^{27}$ reported that $24 \%$ of the participants failed the monocular amplitude testing, and $26 \%$ failed the binocular testing. Both studies did not specify a diagnosis of AI. Although these 
TABLE 2: Summary of studies on accommodative insufficiency with prevalences.

\begin{tabular}{|c|c|c|c|c|c|}
\hline Author/s and year of study & Country of study & Study setting & Age (years) & Sample size & Prevalence (\%) \\
\hline Borsting et al. $(2003)^{14}$ & USA & School setting & $8-15$ & 392 & 17 \\
\hline Marran et al. $(2006)^{15}$ & USA & School setting & 11.5 & 299 & 4.7 \\
\hline Sterner et al. $(2004)^{19}$ & Sweden & School setting & $6-10$ & 76 & 34 \\
\hline Abdi and Rydberg $(2005)^{20}$ & Sweden & School setting & $6-16$ & 120 & 24.2 \\
\hline Abdi et al. $(2008)^{21}$ & Sweden & School setting & $6-16$ & 216 & 11.1 \\
\hline Shin et al. $(2009)^{22}$ & South Korea & School setting & $9-13$ & 114 & 18.3 \\
\hline Dusek et al. $(2010)^{25}$ & Austria & Optometric practice & $6-14$ & 328 & 0.6 \\
\hline Scheiman et al. $(1996)^{17}$ & USA & University clinic & $6-18$ & 1650 & 2.3 \\
\hline Benzoni and Rosenfield $(2012)^{18}$ & USA & University clinic & $5-10$ & 60 & 36 \\
\hline Helveston et al. $(1985)^{16}$ & USA & School setting & $\mathrm{N} / \mathrm{A}$ & 1910 & $7-10$ \\
\hline Wajuihian and Hansraj (2014) & South Africa & School setting & $13-19$ & 65 & 1.6 \\
\hline Moodley $(2008)^{27}$ & South Africa & School setting & $6-13$ & 264 & 24 \\
\hline Metsing and Ferreira $(2012)^{26}$ & South Africa & School setting & $8-13$ & 112 & 10 \\
\hline
\end{tabular}

N/A, not available.

Note: Please see the full reference list of the article, Wajuihian So, Hansraj R. A review of non-strabismic accommodative and vergence anomalies in school-age children. Part 2: Accommodative anomalies. Afr Vision Eye Health. 2015;74(1), Art. \#33, 7 pages. http://dx.doi.org/10.4102/aveh.v74i1.33, for more information.

TABLE 3: Summary of studies on accommodative excess.

\begin{tabular}{|c|c|c|c|c|c|}
\hline Authors/year of study & Country of study & Study setting & Age (years) & Sample size & Prevalence (\%) \\
\hline Shin et al. $(2009)^{22}$ & South Korea & School setting & $9-13$ & 114 & 3.7 \\
\hline Scheiman et al. $(1996)^{17}$ & USA & University clinic & $6-18$ & 1650 & 1.8 \\
\hline Wajuihian and Hansraj (2014) ${ }^{23}$ & South Africa & School setting & $13-19$ & 65 & 0 \\
\hline
\end{tabular}

Note: Please see the full reference list of the article, Wajuihian SO, Hansraj R. A review of non-strabismic accommodative and vergence anomalies in school-age children. Part 2: Accommodative anomalies. Afr Vision Eye Health. 2015;74(1), Art. \#33, 7 pages. http://dx.doi.org/10.4102/aveh.v74i1.33, for more information.

studies provided relevant information on accommodative functions in primary school children, the non-random selection of participants in both studies and the retrospective design (which has minimal control for bias) of the study by Moodley ${ }^{27}$ and the relatively small sample size in the study by Metsing and Ferreira ${ }^{26}$ may limit generalisation of the findings from both studies. The findings from studies on accommodative insufficiency are summarised in Table 2.

\section{Accommodative excess}

AE describes an anomaly where an individual has difficulty in relaxing accommodation. ${ }^{8,28}$ In $\mathrm{AE}$, the individual has a greater accommodative response than the stimulus (or demand) requires, with a tendency to bring the primary focal point abnormally close to the eye. ${ }^{31}$ AE has been used interchangeably with ciliary spasm, hyper-accommodation, accommodative spasm, pseudo-myopia and spasms of the near reflex. ${ }^{8,31} \mathrm{AE}$ is a more subtle type of accommodative spasm and is not synonymous with pseudo-myopia although it can induce pseudo-myopia. ${ }^{8,30}$ The direct diagnostic signs for $\mathrm{AE}$ include difficulty clearing $2 \mathrm{D}$ with monocular accommodative facility and a lead on MEM retinoscopy, whilst the indirect diagnostic signs include reduced NRA, difficulty clearing $2 \mathrm{D}$ with binocular accommodative facility, fused cross cylinder findings, low base-in-to-blur findings at near and esophoria at near. . $^{8,1}$

Previous studies on AE and AIF are essentially the studies that reported on $\mathrm{AI}$; therefore a review of the details of the study methods is omitted here owing to space constraints. $\mathrm{AE}$ has been less frequently reported than AI. In Dusek et al.'s study ${ }^{25}$ on Austrian schoolchildren, no participant had AE. Scheiman et al. ${ }^{17}$ reported a $1.8 \%$ prevalence of AE, which was more higher in female $(2.6 \%)$ than in male $(1.1 \%)$ subjects. A higher prevalence of $3.7 \%$ was reported by Shin et al. ${ }^{22}$ on a population of elementary school children in South Korea. The findings from studies on AE are summarised in Table 3.

\section{Accommodation infacility}

Accommodative infacility (AIF), also referred to as inertia or tonus, ${ }^{12,28}$ is an accommodative anomaly where an individual has difficulty in shifting focus quickly and efficiently from the chalkboard to the desk and back. ${ }^{8,9}$ As with the studies reviewed on AE, only a few studies reported on AIF. The direct diagnostic signs in AIF include difficulty clearing the $-2 \mathrm{D}$ or $2 \mathrm{D}$ lenses with monocular accommodative facility, reduced relative accommodation findings and low base-out and base-in-to-blur findings. ${ }^{8}$ The study by Scheiman et al. ${ }^{17}$ on American schoolchildren aged between 6 and 18 years found a prevalence of $1.2 \%$ for AIF, which increased with age. The criteria used to diagnose AIF were not specified. Dwyer reported a higher prevalence $(5 \%)$ of AIF in a population of Australian schoolchildren aged between 7 and 18 years. Shin et al. ${ }^{22}$ reported a much higher prevalence, of $13.4 \%$, in South Korean schoolchildren aged between 9 and 13 years. In Daum, ${ }^{12} 12.3 \%$ of the participants selected from a clinical population of patients with accommodative problems had AIF. Similarly, Metsing and Ferreira reported that $12.3 \%$ of the participants in their study had poor AF whereas Moodley ${ }^{27}$ reported that a much higher proportion $(30 \%)$ of the study participants had poor binocular AF. Given that the studies by Metsing and Ferreira ${ }^{26}$ and Moodley ${ }^{27}$ were conducted on populations of South African schoolchildren and on similar 
TABLE 4: Summary of studies on accommodative infacility

\begin{tabular}{|c|c|c|c|c|c|}
\hline Authors/year of study & Country of study & Study setting & Age (years) & Sample size & Prevalence $(\%)$ \\
\hline Shin et al. $(2009)^{22}$ & South Korea & School setting & $9-13$ & 114 & 13.4 \\
\hline Dwyer $(1992)^{24}$ & Australia & Optometry practice & $7-18$ & 144 & 5 \\
\hline Daum $(1983)^{12}$ & USA & Optometry practice & 18.5 & 114 & 12.3 \\
\hline Scheiman et al. $(1996)^{17}$ & USA & University optometry clinic & $6-18$ & 1650 & 1.2 \\
\hline Wick and Hall $(1987)^{11}$ & USA & School setting & $6-12$ & 200 & 53 \\
\hline Wajuihian and Hansraj (2014) & South Africa & School setting & $13-19$ & 65 & 1.6 \\
\hline Moodley $(2008)^{27}$ & South Africa & School setting & $6-13$ & 264 & 30 \\
\hline Metsing and Ferreira $(2012)^{26}$ & South Africa & School setting & $8-13$ & 112 & 12.3 \\
\hline
\end{tabular}

Note: Please see the full reference list of the article, Wajuihian SO, Hansraj R. A review of non-strabismic accommodative and vergence anomalies in school-age children. Part 2: Accommodative anomalies. Afr Vision Eye Health. 2015;74(1), Art. \#33, 7 pages. http://dx.doi.org/10.4102/aveh.v74i1.33, for more information.

TABLE 5: Summary of range of prevalence for all accommodative anomalies.

\begin{tabular}{ll}
\hline Anomaly & Prevalence range (\%) \\
\hline $\begin{array}{l}\text { Accommodative insufficiency in non-clinical setting } \\
\text { (regardless of criteria) }\end{array}$ & $4.3-34$ \\
$\begin{array}{l}\text { Accommodative insufficiency in non-clinical setting } \\
\text { (defined with multiple criteria) }\end{array}$ & $4.7-18.3$ \\
$\begin{array}{l}\text { Accommodative insufficiency (regardless of criteria) in } \\
\text { clinical setting }\end{array}$ & $0.6-10$ \\
$\begin{array}{l}\text { Accommodative insufficiency (defined with multiple } \\
\text { criteria)) in clinical setting }\end{array}$ & $2.3-9.2$ \\
$\begin{array}{l}\text { Poor accommodative amplitude } \\
\text { Accommodative excess regardless of study setting and } \\
\text { criteria }\end{array}$ & $7-36$ \\
$\begin{array}{l}\text { Accommodative infacility regardless of study setting and } \\
\text { using multiple criteria }\end{array}$ & $1.2-13.4$ \\
$\begin{array}{l}\text { Poor accommodative facility regardless of study setting } \\
\text { and using the single criterion of 'failed accommodative } \\
\text { facility' testing }\end{array}$ & $5-53$ \\
\hline
\end{tabular}

age ranges, the differences in the prevalence reported may be related to the subjectiveness of AF testing, which is often difficult for young children to comprehend and respond to appropriately. Wick and Hall ${ }^{11}$ reported the highest prevalence of $53 \%$ for poor binocular $\mathrm{AF}$ in a population of schoolchildren in the USA. In a pilot study comprising 65 high school learners, Wajuihian and $\mathrm{Hansraj}^{23}$ reported a prevalence estimate of $1.6 \%$ for AI. The findings from studies on accommodative infacility are summarised in Table 4, and a summary of the prevalences of all accommodative anomalies is given in Table 5 .

\section{Discussion}

In the present article, the prevalence of accommodative anomalies was reviewed. The findings from the reviewed studies are summarised in Tables 2-4. Although the reviewed studies provide workable prevalence estimates of accommodative anomalies, the vast variations in the clinical measurement techniques and findings from reviewed studies make it difficult to interpret and apply the findings consistently. The possible sources of variation and limitations of previous studies are outlined. It is hoped that identifying the problems will guide improvement of future studies.

\section{Sources of variation}

The methodological sources of variations identified from previous studies include:

- differences in instrumentation and techniques

- differences in classification criteria and the number of diagnostic signs
- differences in data analysis methods

- differences in the type of population studied (clinical/ non-clinical)

- representativeness of the populations (probability/nonprobability sampling methods)

- inter-examiner variations

- participant factors that include age as well as the fact that some techniques such as accommodative facility, amplitude of accommodation, relative accommodation, and fusional vergence measurements may affect findings across studies

- changes in the nature of near point tasks may be a source of variation in findings across studies.

\section{Limitations of previous studies}

- Most studies failed to detail their sampling strategies and how sample sizes were derived, whilst some studies used small sample sizes with a consequent low statistical power. Specifically, sample size determination is important to ensure that the study has a good chance of detecting a statistically significant result if the effect is true that minimises type II error (chances of missing a significant difference), ${ }^{85,86}$ thereby enhancing the generalisability of study findings.

- The eligibility criteria (inclusion and exclusion) that are important in understanding the sample studied, and interpreting and assessing the study validity, ${ }^{23,45}$ were not indicated in some studies.

- In some studies, samples were derived from clinical or conveniently selected samples. Although some study settings can only permit retrospective and convenience sampling methods which are relevant in providing baseline information, a drawback of such studies is that the reported prevalence may be biased as the study design did not allow for control of bias. In most cases, conveniently selected samples would not permit probability sampling, and most statistical formulas are not designed for samples selected using convenience sampling.

- Another limitation is that some studies failed to either indicate the diagnostic criteria or specify the clinical signs used to define the outcome variable. Failure to report these parameters may affect the internal validity of a study and limit interpretations of study findings. Also, it is important to note that it is rare to find a study without inherent limitations. Most reviewed studies 
failed to indicate the limitations of the studies. Indicating the limitations of a study will enable improvements to subsequent studies.

- Invariably, some conditions such as AI have been studied extensively whereas other anomalies such as AE and AI were less studied.

- Overall, there are limited data regarding how ethnicity, race, age, gender, geographic location and socio-economic status influence accommodative anomalies. Similarly, there are limited studies on black African populations.

- No study could be found that reported on the syndromes of accommodation anomalies in any black South African population. Remarkably, there are also no available studies conducted on high school children in a school setting. The high school populations are particularly relevant to study as they are vulnerable to accommodative anomalies because of the relatively high near task demands they are exposed to, given that near tasks increase with higher school grade levels and as children prepare for tertiary education, which has even greater near tasks demand.

\section{Significance and limitations of the present review}

A major strength of the present article is an extensive and upto-date review of the available literature on accommodative anomalies in school-age children. A possible limitation is that only studies reported in the English language were retrieved; it is possible that articles reported in other languages were omitted. Secondly, only published papers were reviewed; credible unpublished studies might have been omitted. Despite the outlined limitations, the present review is significant and has important implications and applications in optometric education, clinical practice, research and health policy planning.

\section{Recommendations for future studies}

The following recommendations are aimed at improving future research on accommodative (and vergence) anomalies:

- To minimise variations in classifications and investigative techniques, international expert bodies similar to the Convergence Insufficiency and Reading Study Group and the Convergence Insufficiency Treatment Trial groups should be initiated to stipulate a standardised study design (common definitions, measuring techniques and diagnostics criteria, statistical analyses and reporting methods) for accommodative anomalies. Such criteria should be used as a benchmark to access credibility of subsequent studies. Consistent diagnostic techniques and criteria are important, considering how they influence diagnosis and treatment.

- Accommodative anomalies are prevalent in schoolage populations and, given their clinical significance, it is important that optometrists and other eye care professionals consider it mandatory to evaluate, diagnose and treat these anomalies in all settings. Continuing research by professionals should also be considered of major importance.
- Further research is needed to investigate whether the prevalence of accommodative anomalies varies by ethnicity, race, age, sex, geographic location or socioeconomic status. Data can be gathered via collaborations between international researchers.

- Studies on high school populations will be relevant.

- It is important that authors adequately detail all testing protocols to enable replication of the study by other researchers.

- Studies with larger sample sizes using random sampling would yield more valid results.

\section{Summary and conclusion}

A summary of the reviewed studies is included in Tables 2-4. Irrespective of whether single or multiple clinical signs are used to classify accommodative anomalies, the present review revealed that these anomalies are prevalent in all settings in school-age populations (Tables 2-5). The lack of uniform diagnostic protocols and classification criteria has complicated comparing prevalence estimates from different studies. The present review has provided a workable prevalence range for accommodative anomalies and highlighted some methodological concerns in previous studies. The findings of the present review have implications and applications for practice, education, research and policy administration. Given that treatment regimens are based on appropriate measurement and diagnosis, it is important to establish a standardised worldwide diagnostic protocol and criteria with the ultimate goal of optimum patient care. Continued discourse, which will further enhance our understanding of accommodative anomalies, is certainly needed, given that the negative consequences of untreated accommodative anomalies are numerous. Optometry plays a major role in the detection, assessment, and management of visual anomalies in children, and the optometrist is often one of the healthcare professionals whom parents consult when a child performs poorly academically.

\section{Acknowledgements}

We are grateful to Mr P. Mashige and Professor B.M. Zungu for reviewing the initial drafts of the manuscript.

\section{Competing interests}

The authors declare that they have no financial or personal relationships which may have inappropriately influenced them in writing this article.

\section{Authors' contributions}

S.O.W. (University of KwaZulu-Natal) wrote the manuscript. R.H. (University of KwaZulu-Natal) contributed intellectually overall and played a supervisory role.

\section{References}

1. Garzia R. The relationship between visual efficiency problems and learning In Scheiman $M$, Rouse $M$, editors. Optometric management of learning-related vision problems. St. Louis: Mosby; 2006; pp. 209-280. 
2. Hoffman LG, Rouse M. Referral recommendations for binocular function and/or developmental perceptual deficiencies. J Am Optom Assoc. 1980;51:119-125.

3. Bodack M, Chun I, Krumholtz I. An analysis of vision screening data from New York City public schools. Optometry. 2010;81:476-484. http://dx.doi.org/10.1016/j. optm.2010.05.006

4. Cacho-Martínez P, García-Muñoz A, Ruiz-Cantero MT. Do we really know the prevalence of accommodative and nonstrabismic binocular dysfunctions? J Optom. 2010;3:185-197. http://dx.doi.org/10.1016/S1888-4296(10)70028-5

5. Birnbaum M. Symposium on near point visual stress. Introduction. Am J Optom Physiol Opt. 1985;62:361-364. http://dx.doi.org/10.1097/00006324-19850600000001

6. Duane A. Anomalies of accommodation clinically considered. Arch Ophthal. 1916;45:124-136.

7. Duane A. Studies in monocular and binocular accommodation with their clinical application. Am J Ophthalmol. 1922;5:565-577. http://dx.doi.org/10.1016/ S0002-9394(22)90793-7

8. Scheiman M, Wick B. Clinical management of binocular vision: Heterophoric, accommodative and eye movement disorders. 3rd edn. Philadelphia, PA: JB Lippincott; 2008

9. Sterner B, Gellerstedt M, Sjöström A. Accommodation and the relationship to subjective symptoms with near work for young school children. Ophthal Physiol Opt. 2006;26:148-155. http://dx.doi.org/10.1111/j.1475-1313.2006.00364.x

10. Von Noorden G, Campos EC. Binocular vision and ocular motility, theory and management of strabismus. 6th edn. Missouri: Mosby Inc.; 2002.

11. Wick B, Hall P. Relation among accommodative facility, lag, and amplitude in elementary school children. Am J Optom Physiol Opt. 1987;64:593-598. http:// dx.doi.org/10.1097/00006324-198708000-00005

12. Daum KM. Accommodative dysfunction. Doc Ophthalmol. 1983;55:177-198. $\mathrm{http}: / / \mathrm{dx}$.doi.org/10.1007/BF00140808

13. American Optometric Association. Optometric clinical practice guidelines: Care of the patient with accommodative and vergence dysfunctions. c2013 [cited 15 January 2013]. Available from: http://www.aoa.org/documents/CPG-20

14. Borsting E, Rouse MW, Deland PN, et al. Association of symptoms and convergence and accommodative insufficiency in school-age children. Optometry. 2003;74:25-34.

15. Marran LF, De Land PN, Nguyen AL. Accommodative insufficiency is the primary source of symptoms in children diagnosed with convergence insufficiency. Optom Vis Sci. 2006;83:281-289. http://dx.doi.org/10.1097/01. opx.0000216097.78951.7b

16. Helveston E, Weber J, Miller K, Robertson K. Visual function and academic performance. Am J Ophthalmol. 1985;99:346-555. http://dx.doi.org/10.1016/ 0002-9394(85)90368-X
17. Scheiman M, Gallaway M, Coulter R, Reinstein F, Ciner E. Prevalence of vision and ocular disease conditions in a clinical pediatric population. J Am Optom Assoc. 1996;67:193-202.

18. Benzoni JA, Rosenfield M. Clinical amplitude of accommodation in children between 5 and 10 years of age. Optom Vis Dev. 2012;43:109-114.

19. Sterner B, Gelleerstedt M, Sjostrom A. The amplitude of accommodation in 6-10-year-old children - not as good as expected. Ophthal Physiol Opt. 2004;24:246-251. http://dx.doi.org/10.1111/j.1475-1313.2004.00201.x

20. Abdi S, Rydberg A. Asthenopia in schoolchildren, orthoptic and ophthalmologica findings and treatment. Doc Ophthalmol. 2005;111:65-72. http://dx.doi.org/ $10.1007 / \mathrm{s} 10633-005-4722-4$

21. Abdi S, Lennerstrand G, Pansell T, Rydberg A. Orthoptic findings and asthenopia in a population of Swedish schoolchildren aged 6 to 16 years. Strabismus. 2008;16:47-55. http://dx.doi.org/10.1080/09273970802020243

22. Shin HS, Park SC, Park CM. Relationship between accommodative and vergence dysfunction and academic achievement for primary school children. Ophthal Physiol Opt. 2009;29:615-624. http://dx.doi.org/10.1111/j.1475-1313.2009. 00684.x

23. Wajuihian SO, Hansraj R. Near vision anomalies in a population of black high school children in South Africa: A pilot study. S Afr Optom. 2014;73:21-32.

24. Dwyer $P$, Wick $B$. The influence of correction upon disorders of vergence and accommodation. Optom Vis Sci. 1995;72:224-232. http://dx.doi.org/10.1097/ 00006324-199504000-00002

25. Dusek W, Piersionek BK, McClelland JF. A survey of visual functions in an Austrian population of school-age children with reading and writing difficulties. BMC Ophthalmol. 2010;10:1-10. http://dx.doi.org/10.1186/14712415-10-16

26. Metsing IT, Ferreira JT. Accommodation and vergence status among the 3rd and 4th graders in a mainstream school in Gauteng. S Afr Optom. 2012;71: 22-31.

27. Moodley VR. Amplitude, facility and accuracy of accommodation in primary school population. S Afr Optom. 2008;67:147-154

28. Griffin JR, Grisham JD. Binocular anomalies: Diagnosis and vision therapy. 4th edn Boston, MA: Butterworth-Heinemann; 2002.

29. Chen AH, Abidin AHZ. Vergence and accommodation systems in Malay primary school children. J Biomed Sci. 2002;9:9-15.

30. London R. Amplitude of accommodation. In: Eskridge JB, Amos JF, Bartlett JD, editors. Clinical procedures in optometry. Philadelphia, PA: JB Lippincott; 1991.

31. Rustein RP, Daum KM, Amos JF. Accommodative spasm: A study of 17 cases. J Am Optom Assoc. 1988;59:527-538. 